\title{
INCIDÊNCIA DE LOMBALGIA NOS ALUNOS DO OITAVO PERÍODO DO CURSO DE ODONTOLOGIA DA UNIG CAMPUS V E A SUA REAL INTERFERÊNCIA NA QUALIDADE DE VIDA
}

\author{
Rhobert Gomes Frauches ${ }^{1}$; Walace Érick de Medeiros Moura² ; Júlio Corrêa \\ Campos $^{2}$; Silvani Barreto Assumpção Cardoso ${ }^{2}$ \& Juliano Silva França ${ }^{3}$
}

http://dx.doi.org/10.18571/acbm.004

\section{RESUMO}

Esse estudo teve como objetivo analisar de maneira quantitativa o índice de lombalgia nos acadêmicos do oitavo período do curso de odontologia da UNIG campus $\mathrm{V}$ em Itaperuna RJ, e avaliar a qualidade de vida dos mesmos, a fim de chegar a esses objetivos foi aplicado um questionário contendo questões como, sexo, idade, altura, peso, hábitos de vida, tipos de instrumentos usados durante as intervenções odontológicas, se sentem dores constantes ou não, e um quadro para relatar com precisão onde se localiza essa dor, também foram aplicados mais dois instrumentos de auto-avaliação são eles o questionário de Roland-Morris com o objetivo de avaliar a real gravidade e o nível de incapacidade física causada pela lombalgia, e o questionário SF36 para avaliar o impacto causado pela lombalgia na qualidade de vida dos alunos estagiários, também foram realizadas tomadas fotográficas a fim de analisar as posturas adotadas por esses alunos estagiários durante o atendimento odontológico, essas fotos foram analisadas por um profissional fisioterapeuta. A lombalgia é uma das principais causas de afastamento do trabalho para tratamento médico, e um dos principais fatores que levam o profissional da odontologia a aponsentar-se precocemente. De acordo com a interpretação dos dados alcançados nessa pesquisa de campo, fica concluído que a lombalgia não tem interferido de maneira significativa nas atividades de vida diárias dos acadêmicos de odontologia.

Palavras chave: Lombalgia, acadêmicos de odontologia, Qualidade de vida

\begin{abstract}
This study aimed to analyze quantitatively the rate of lower back pain in students in the eighth period of-the dentistry course of the UNIG campus V Itaperuna RJ, and evaluate their quality of life. In order to reach these objectives a questionnaire was used with questions such as gender, age, height, weight, lifestyle, types of instruments used during dental interventions, whether they feel constant pain or not, and a framework to accurately report where the pain is. In addition two self-assessment tools were used: the Roland-Morris questionnaire, to evaluate the actual severity and level of disability caused by back pain, and the SF-36 to assess the impact of Lower Back Pain on the quality of life for student trainees. Photographs were also taken to examine the posture adopted by these students trainees during dental care, and these pictures were analyzed by a professional physiotherapist. Lower back pain is a major cause of absence from work for medical treatment, and one of the main factors leading to dental professionals taking early retirement. According to the interpretation of data obtained in this research field, the conclusion has been reached that lower back pain has not interfered significantly in the daily activities of dental students.
\end{abstract}

Keywords: Lower back pain, dental students, quality of life

1 - Fisioterapeuta

2 - Professor do Curso de Fisioterapia da Universidade Iguaçu - Campus V

3 - Coordenador do Curso de Fisioterapia da Universidade Iguaçu - Campus V

* autor para correspondência: rgomesfrauches@gmail.com 
Rhobert Gomes Frauches; Walace Érick de Medeiros Moura; Júlio Corrêa Campos; Silvani Barreto Assumpção Cardoso \& Juliano Silva França

Incidência de Lombalgia nos Alunos do Oitavo Período do Curso de Odontologia da UNIG Campus V e a Sua Real Interferência na Qualidade de Vida

\section{Introdução}

A lombalgia é a dor na região lombar, e possui varias causas, algumas delas são: congênitas, inflamatórias, degenerativas, tumorais, infecciosas e principalmente mecânico-posturais. Para Andrade, et al, (2005), esse tipo de dor atinge diversos profissionais em países industrializados, entre eles o profissional que mais preocupa é o Cirurgião Dentista, Casarin \& Caria (2008) citam que cerca de 60\% dos cirurgiões dentistas, relatam sofrer algum tipo de lesão musculoesquelética, sendo o pescoço, ombro, membros superiores e principalmente a região lombar os mais afetados.

Algumas pesquisas apontam que os Cirurgiões Dentistas se afastam das suas atividades laborais com certa frequiência para o tratamento médico de dores lombares, e que, esse tipo de algia tem levado esse profissional a se aposentar precocemente. Segundo Bachiega (2009), desde a década de 50 que alguns estudos sobre Distúrbios Osteomusculares Relacionados ao Trabalho (DORT) em Cirurgiões Dentistas vêm sendo realizados, e hoje pesquisas colocam esse profissional entre os primeiros lugares em afastamento do trabalho, seja temporário ou permanente.

Esse tipo de problema é proveniente das más posturas adotadas por esses profissionais durante os atendimentos que eles prestam, pois permanecem sentados durante horas realizando movimentos repetitivos, forçando as estruturas articulares e musculares, Graça, et al, (2006) citam que quando o cirurgião dentista realiza suas atividades de trabalho adotando postura sentada, ele tende a desenvolver complicações cardiovasculares, respiratórias, circulatórias em membros inferiores e principalmente lombalgia.

De fato é preocupante como a lombalgia vem acometendo os Cirurgiões Dentistas e interferindo nas atividades de vida diária dos mesmos, frente a essas informações esse trabalho teve como objetivo analisar de maneira quantitativa a incidência de lombalgia em alunos do oitavo período do curso de odontologia da UNIG campus V e verificar qual é a real interferência da lombalgia na qualidade de vida desses acadêmicos.

\section{Material e Métodos}

A coleta de dados ocorreu na sala de aula dos alunos do oitavo período do curso de odontologia da UNIG - Campus V em Itaperuna-RJ, no dia 25 de novembro de 2010. No qual, foi aplicado um questionário validado de identificação para caracterizar a população estudada, também, foi aplicado o questionário de Roland-Morris com o objetivo de avaliar o nível de incapacidade física causada pela lombalgia, segundo Ocarino, et al, (2009), o questionário de Roland-Morris avalia o impacto da lombalgia no desempenho funcional, ele é constituído de 24 itens que exemplificam as consequiências da dor lombar, esse questionário apresenta uma alta confiabilidade, é de fácil apresentação e possuí um escore padronizado. Foi utilizado, também, o questionário SF-36 que foi o terceiro e o último instrumento de auto-avaliação a ser aplicado na referida turma. Ele avalia a qualidade de vida através de oito parâmetros, Soares (2011) afirma que o questionário SF-36 é de fácil manejo e compreensão, ele analisa de forma quantitativa o nível da qualidade de vida da população a ser estudada. A tomada fotográfica foi realizada alguns dias após a coleta de dados, na Clínica Escola de Odontologia da Universidade, para observar a postura adotada pelos alunos durante os procedimentos odontológicos e para avaliação ergonômica entre profissionais e equipamentos utilizados. 
Rhobert Gomes Frauches; Walace Érick de Medeiros Moura; Júlio Corrêa Campos; Silvani Barreto Assumpção Cardoso \& Juliano Silva França

Incidência de Lombalgia nos Alunos do Oitavo Período do Curso de Odontologia da UNIG Campus V e a Sua Real Interferência na Qualidade de Vida

A amostra foi composta por 16 acadêmicos (n=16), ou seja, $40 \%$ do total de 40 alunos do oitavo período do curso de odontologia da Universidade, que realizam seus atendimentos na maior parte do tempo sentados. A idade desses estudantes está entre 21 a 32 anos, o peso varia de 51 a $104 \mathrm{Kg}$, e estatura de 156 a $185 \mathrm{~cm}$. Não houve a necessidade dos estudantes envolvidos na pesquisa assinarem um termo de autorização, uma vez que o sigilo dos mesmos foi mantido.

A análise da casuística se deu com o auxilio do programa Excel for Windows da Microsoft Inc e do pacote computacional Statistic (versão 7.0) da Stat Soft Inc.

\section{Resultados}

Para chegar aos objetivos propostos, esta pesquisa utilizou alguns instrumentos para coleta de dados, como questionários e câmera fotográfica, cada qual com sua função e propósito.

O questionário de Identificação foi utilizado para caracterizar a população estudada fornecendo informações como: idade, sexo, altura, peso, horas de trabalho e intervalos, outras atividades associadas, constância e localização de dores, consumo de álcool e cigarros, prática de atividades físicas. Esse questionário permitiu identificar o grupo de acadêmicos da odontologia que sentem dor nas partes baixas das costas, ou seja, sofrem com a lombalgia.

O questionário de Roland-Morris foi utilizado para que através de 24 alternativas, os alunos entrevistados pudessem demonstrar se conseguiriam executar suas tarefas diárias com êxito ou com dificuldade, já que alguns possuem um quadro álgico na região lombar, mesmo este sendo pequeno. Esse questionário permitiu analisar a gravidade existente e o nível de incapacidade física causada pela dor lombar em cada acadêmico entrevistado.

Considerando que tivemos dois grupos, ou seja, COM e SEM dor lombar, foi possível encontrar os escores expostos na Tabela 1.1

\begin{tabular}{ccc}
\hline Quant. de afirmações & $\begin{array}{c}\text { COM dor } \\
(\mathrm{n}=6)\end{array}$ & $\begin{array}{c}\text { SEM dor } \\
(\mathrm{n}=10)\end{array}$ \\
0 & - & 1 \\
1 & 4 & 3 \\
2 & - & 2 \\
3 & - & - \\
4 & - & 1 \\
5 & 2 & - \\
6 & - & - \\
7 & - & 1 \\
8 & - & 1 \\
9 & 14 & 35 \\
\hline
\end{tabular}

Tabela 1.1 - Escore obtido no questionário de Roland-Morris

Os escores do questionário de Roland-Morris foram obtidos através da multiplicação do número de pessoas pelo número de afirmações, depois os resultados encontrados são somados, de acordo com cada grupo, por exemplo, no grupo COM DOR, 4 pessoas $\times 1$ afirmação $=4 ; 2$ pessoas x 5 afirmações $=10,4+10=14$.

De acordo com os escores obtidos por meio das afirmações, foi possível encontrar a média para cada grupo (Tabela1.2), essas médias são alcançadas com a divisão da 
Rhobert Gomes Frauches; Walace Érick de Medeiros Moura; Júlio Corrêa Campos; Silvani Barreto Assumpção Cardoso \& Juliano Silva França

Incidência de Lombalgia nos Alunos do Oitavo Período do Curso de Odontologia da UNIG Campus V e a Sua Real Interferência na Qualidade de Vida

somatória de itens marcados pelo numero de indivíduos, como por exemplo: 14 (itens) / 6 (indivíduos) $=2,33$.

COM dor

$\mathrm{RM}$
SEM dor

$3,5^{*} \pm 3,09^{* *}$

*média

**desvio-padrão

Tabela 1.2 - Média obtida a partir do questionário de Roland-Morris

No escore de função é considerado que quando mais perto de 24 pontos pior é a capacidade funcional, a média apresentada pelo grupo COM dor nesse estudo, foi de $2,33 \pm 2,06$, o quadro álgico apresentada por esse grupo de acadêmicos, tem apresentado um grau pequeno, não prejudicando a execução de suas atividades de vida diárias.

O questionário SF-36 foi o instrumento que nos possibilitou verificar o impacto causado pela lombalgia na vida dos estagiários de odontologia, uma vez que ele permitiu avaliar a qualidade de vida em vários aspectos da população a ser estudada.

Os dados de qualidade de vida foram tratados segundo proposto pelo questionário SF-36, conforme Prado (2006). Primeiro foi estabelecido a correspondência de cada item ou questão à sua respectiva escala. Conforme Tabela 1.3.

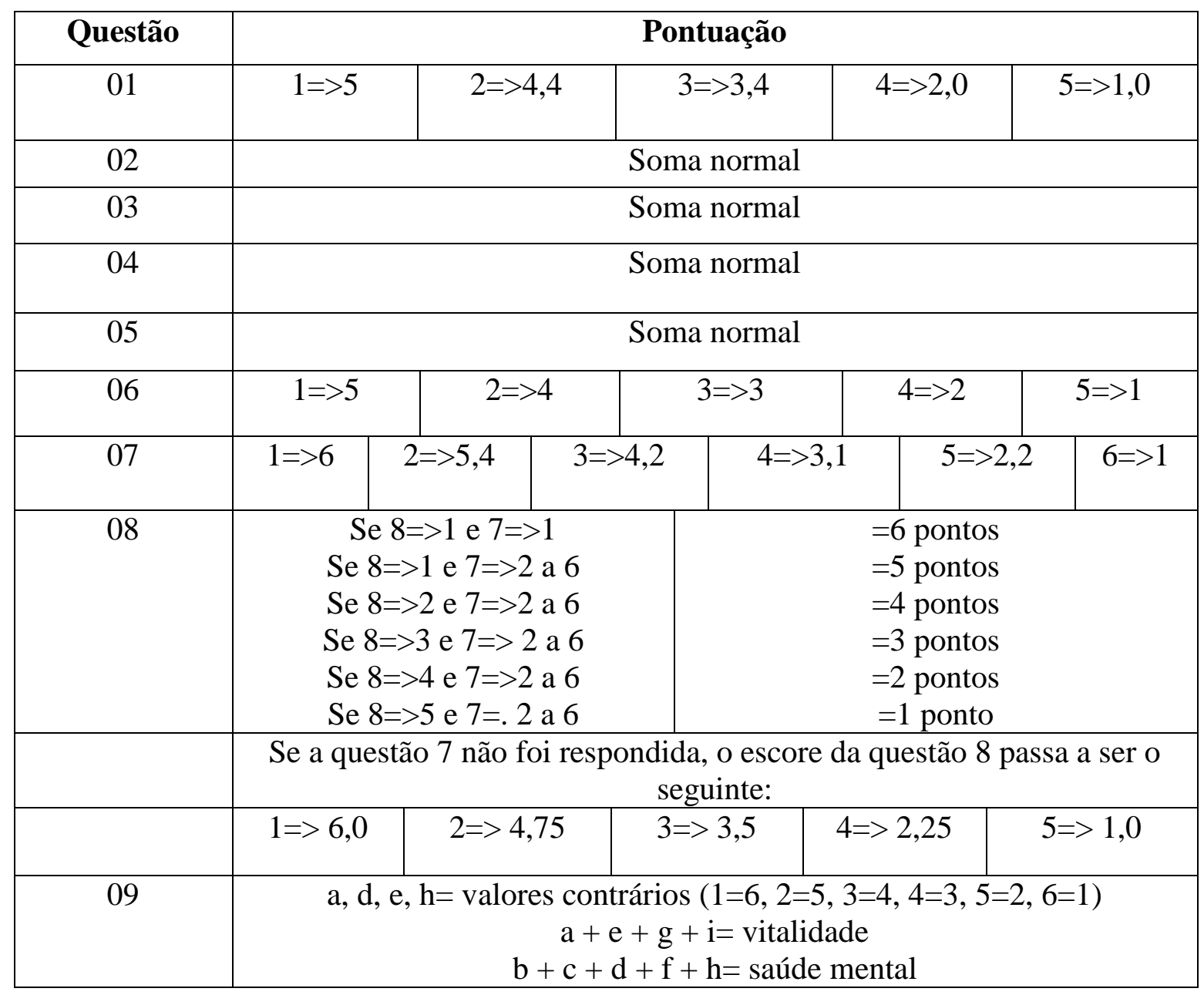


Rhobert Gomes Frauches; Walace Érick de Medeiros Moura; Júlio Corrêa Campos; Silvani Barreto Assumpção Cardoso \& Juliano Silva França

Incidência de Lombalgia nos Alunos do Oitavo Período do Curso de Odontologia da UNIG Campus V e a Sua Real Interferência na Qualidade de Vida

\begin{tabular}{|c|c|}
\hline 10 & Soma normal \\
\hline 11 & a, c= valores normais \\
& b, d= valores contrários $(1=5,2=4,3=3,4=2,5=1)$ \\
\hline
\end{tabular}

Tabela 1.3 - Pontuação do questionário SF-36. Fonte: (Prado, 2006)

Dez itens foram recodificados numa outra etapa, onde apareceram invertidos na pontuação ou desiguais em número de opção de uma mesma escala (Tabela 1.3). Exemplo escala "Dor", composta pelos itens 7 e 8 . O item 7, é formado por seis alternativas de respostas, numeradas de 1 a 6 , o item 8 , é formado por cinco alternativas numeradas de 1 a 5 . Foi feita a uniformização para 6 alternativas em ambas questões.

Com o objetivo de alcançar um alinhamento de valor em todas as questões, atribuíram um escore para cada alternativa, o escore com o valor maior corresponde então a um melhor estado de saúde, e o escore menor corresponde a um pior estado de saúde. Esse fato ocorreu através da realização da recodificação.

No que diz respeito à qualidade de vida, os dados foram computados. Foram somados os escores dos itens de cada escala, os quais correspondiam às respostas dos acadêmicos entrevistados (vide Tabela 1.3). Por exemplo, considerando a escala Estado Geral de Saúde (EGS) foram somados os escores referentes às respostas dos 16 alunos entrevistados aos itens correspondentes à escala EGS, ou seja, os itens 1, 11a, 11b, 11c e 11d (para cada item o respondente atribuiu uma resposta), obtendo um total de 80 respostas, depois tendo os números determinados, foram calculado os respectivos percentuais.

A próxima etapa foi onde se realizou o cálculo do Raw Scale, onde a transformação de cada escore numa escala de 0 a 100 ocorreu. Nesta transformação se converteu o valor mínimo e o máximo para cada item em cada escala e para cada entrevistado. Os escores entre esses valores representam a porcentagem possível de ser alcançada conforme mostra Tabela 1.4.

\begin{tabular}{|c|c|c|c|}
\hline & Questão & Limites & Score \\
\hline $\begin{array}{c}\text { Capacidade funcional } \\
(\mathrm{CF})\end{array}$ & $3(\mathrm{a}+\mathrm{b}+\mathrm{c}+\mathrm{d}+\mathrm{e}+\mathrm{g}+\mathrm{f}+\mathrm{i}+\mathrm{j})$ & $10-30$ & 20 \\
\hline $\begin{array}{c}\text { Aspectos físicos (AF) } \\
\text { Dor (DR) }\end{array}$ & $4(\mathrm{a}+\mathrm{b}+\mathrm{c}+\mathrm{d})$ & $4-8$ & 10 \\
\hline $\begin{array}{c}\text { Estado geral de saúde } \\
(\text { EGS) }\end{array}$ & $1+8$ & $2-12$ & 20 \\
\hline $\begin{array}{c}\text { Vitalidade (VT) } \\
\text { Aspectos sociais (AS) }\end{array}$ & $9(\mathrm{a}+\mathrm{e}+\mathrm{g}+\mathrm{i})$ & $4-24$ & 20 \\
\hline $\begin{array}{c}\text { Aspectos emocionais } \\
(\text { AE) }\end{array}$ & $5+10$ & $2-10$ & 3 \\
\hline
\end{tabular}


Rhobert Gomes Frauches; Walace Érick de Medeiros Moura; Júlio Corrêa Campos; Silvani Barreto Assumpção Cardoso \& Juliano Silva França

Incidência de Lombalgia nos Alunos do Oitavo Período do Curso de Odontologia da UNIG Campus V e a Sua Real Interferência na Qualidade de Vida

\begin{tabular}{|l|c|c|c|}
\hline Saúde mental $(\mathrm{SM})$ & $9(\mathrm{~b}+\mathrm{c}+\mathrm{d}+\mathrm{f}+\mathrm{h})$ & $5-30$ & 25 \\
\hline
\end{tabular}

Tabela 1.4 - Cálculo do Raw Scale (0 a 100). Fonte: (Prado, 2006)

Os passos para o cálculo dos escores transformados foram: primeiro cada uma das escalas (CF, AF, DR, EGS, VT, AS, AE, SM) foram calculadas, depois foram somados os pontos obtidos de cada item relativo à correspondente escala, para cada acadêmico entrevistado.

Os valores mínimos e máximos possíveis foram utilizados para aplicar o seguinte cálculo:

$$
Y i j=\frac{X i j-\operatorname{Min} j}{\operatorname{Max} j-\operatorname{Min} j} 100
$$

Onde: $\mathrm{I}=1,2,3 \ldots 16$ (corresponde ao número de entrevistados),

$\mathrm{J}=\mathrm{CF}, \mathrm{AF}, \mathrm{EGS} . . .($ cada uma das escalas)

$\mathrm{Y} \mathrm{ij}=$ valor transformado do entrevistado I, escala $\mathrm{J}$.

$\mathrm{X}$ ij = valor da escala $\mathrm{J}$, da entrevistada $\mathrm{I}$.

Min $\mathbf{j}$ = valor mínimo possível para escala $\mathbf{J}$.

Max $\mathbf{J}=$ valor Maximo possível para escala $\mathbf{J}$.

Então se obteve a média de cada parâmetro (CF, AS, EGS.....), por grupo, COM DOR $(n=6)$ e SEM DOR $(n=10)$, e de grupo GERAL $(n=16)$.

As fotos abaixo demonstram posturas inadequadas, durante atendimento odontológico, Garcia, et al, (2008), citam que a postura inadequada, expõe esses profissionais a doenças e distúrbios ocupacionais como LER/DORT. A foto 1.1 demonstra a postura que a aluna adotou durante um atendimento, no qual, realiza rotação de tronco caracterizando uma ação extra-bucal para pegar algum objeto de uso odontológico, e não está com as costas apoiadas no encosto da cadeira, forçando assim as estruturas musculares e articulares da região lombar.

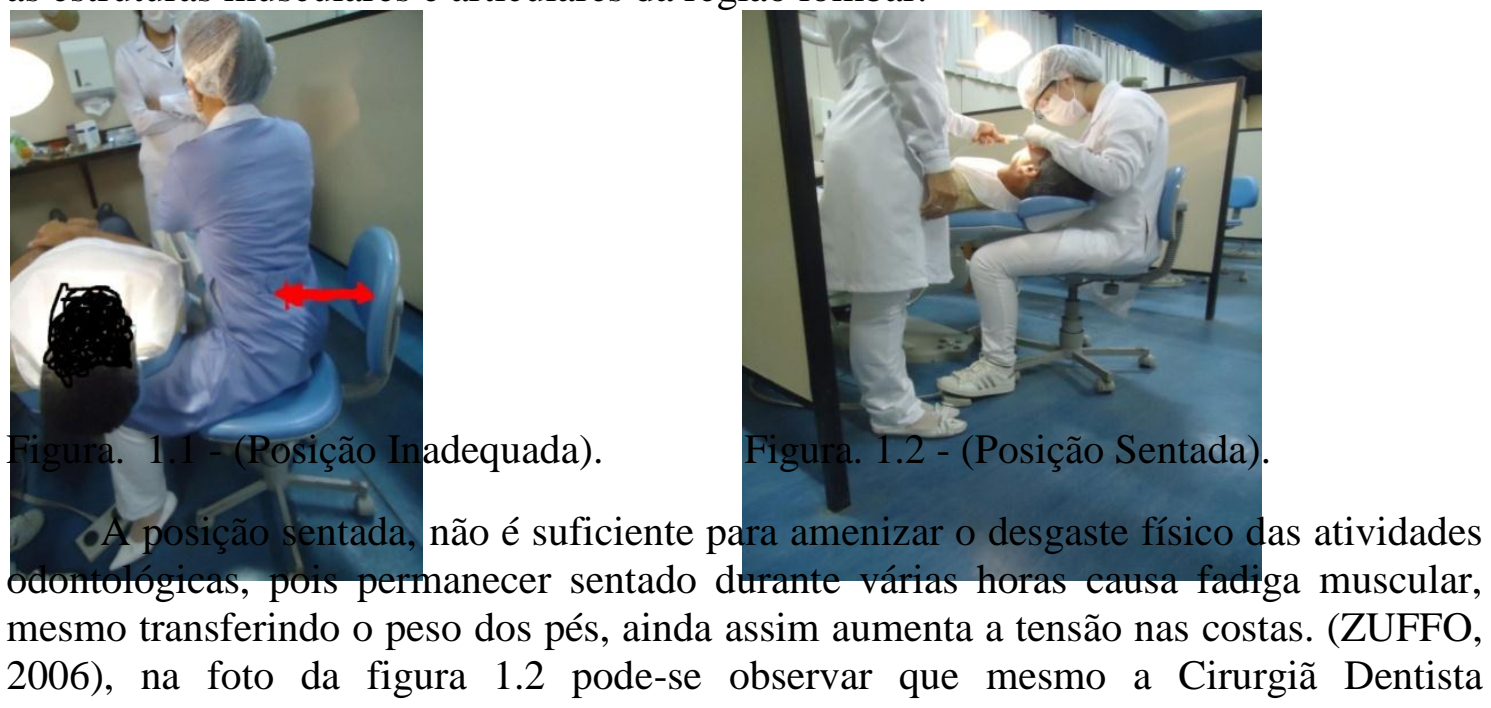


Rhobert Gomes Frauches; Walace Érick de Medeiros Moura; Júlio Corrêa Campos; Silvani Barreto Assumpção Cardoso \& Juliano Silva França

Incidência de Lombalgia nos Alunos do Oitavo Período do Curso de Odontologia da UNIG Campus V e a Sua Real Interferência na Qualidade de Vida

permanecendo sentada durante o atendimento intra-bucal, ela está permanecendo na posição de flexão da coluna cervical, forçando a mesma.

\section{Discussão}

A dor se apresenta de duas maneiras, uma de forma aguda e a outra de forma crônica, a aguda é quando a dor possui duração em até três meses, e a crônica quando permanece por mais de três meses. (LIMA, 2007)

Através dos questionários aplicados, foi possível tabular e trabalhar estatisticamente os dados obtidos. Tornando possível tecer os seguintes comentários.

Com média geral nos oito parâmetros avaliados na qualidade de vida dos estudantes do oitavo período do curso de odontologia foi encontrado: $86 \pm 11,51$ para a capacidade funcional, $85 \pm 24,64$ para aspectos físicos, 71,43 $\pm 22,91$ para dor, 76,68 \pm 17,38 para estado geral de saúde, $56,25 \pm 16,18$ para vitalidade, 70,31 $\pm 24,10$ para aspectos sociais, $62,22 \pm$ 41,53 para aspectos emocionais, $59 \pm 15,70$ para saúde mental.

$\begin{array}{ccc}\begin{array}{c}\text { Estudante em } \\ \text { geral }(n=16)\end{array} & \text { COM dor } & \text { SEM dor } \\ (n=6) & (n=10)\end{array}$

Capacidade funcional (CF) $\quad 86^{*} \pm 11,51^{* *} \quad 89^{*} \pm 11,94 * * \quad 84,5^{*} \pm 11,13^{* *}$

Aspectos físicos (AF)

Dor (DR)

Estado geral de saúde (EGS)

Vitalidade (VT)

Aspectos sociais (AS)

Aspectos emocionais (AE)

Saúde mental (SM)

$$
\begin{array}{rcc}
85^{*} \pm 24,64^{* *} & 90^{*} \pm 22,36^{* * *} & 82,5^{*} \pm 26,48^{* *} \\
71,43^{*} \pm & 70,83^{*} \pm 15,75^{* *} & 71,8^{*} \pm 27,13^{* *} \\
22,91^{* *} & 80^{*} \pm 14,14^{* *} & 74,7^{*} \pm 19,52^{* *} \\
76,68^{*} \pm & \\
17,38^{* *} & & \\
& & \\
56,25^{*} \pm & 50^{*} \pm 7,75^{*} & 60^{*} \pm 19^{* *} \\
16,18^{* *} & & \\
70,31^{*} \pm & 81,25^{*} \pm 17,23^{* *} & 63,75^{*} \pm 25,99^{* *} \\
24,10^{* *} & & \\
62,22^{*} \pm & 79,99^{*} \pm 29,82^{* *} & 53,33^{*} \pm 45^{* *} \\
41,53^{* *} & & \\
59^{*} \pm 15,70^{* *} & 64,66^{*} \pm 19,66^{* *} & 55,6^{*} \pm 12,71^{* *}
\end{array}
$$

Tabela 1.5 - Qualidade de vida dos estudantes de odontologia. (md \pm dp)

Observou-se nos dados descritivos que as médias e desvio padrão para cada uma das dimensões do SF-36 no grupo dos acadêmicos COM DOR estão entre: $89 \pm$ 11,94 para capacidade funcional, $90 \pm 22,36$ para aspecto físico, 70, $83 \pm 15,75$ para dor, $80 \pm 14,14$ para estado geral de saúde, $50 \pm 7,75$ para vitalidade, $81,25 \pm 17,23$ para aspectos sociais, 79,99 $\pm 29,82$ para aspectos emocionais, $64,66 \pm 19,66$ para saúde mental.

No grupo dos alunos SEM DOR os resultados figuram entre: 84,5 $\pm 11,13$ para capacidade funcional, $82,5 \pm 26,48$ para aspecto físico, 71,8 $\pm 27,13$ para dor, 74,7 \pm 19,52 para estado geral de saúde, $60 \pm 19$ para vitalidade, 63,75 $\pm 25,99$ para aspectos sociais, 53,33 \pm 45 para aspectos emocionais, 55,6 \pm 12,71 para saúde mental. 
Rhobert Gomes Frauches; Walace Érick de Medeiros Moura; Júlio Corrêa Campos; Silvani Barreto Assumpção Cardoso \& Juliano Silva França

Incidência de Lombalgia nos Alunos do Oitavo Período do Curso de Odontologia da UNIG Campus V e a Sua Real Interferência na Qualidade de Vida

As pontuações mais altas relatam um melhor estado de saúde e as pontuações mais baixas denotam um pior estado de saúde.

\section{Conclusão}

Apesar dos alunos do oitavo período estarem no inicio da carreira profissional esse estudo demonstrou que existem acadêmicos que assumem posturas inadequadas durante o atendimento odontológico e que isso reflete em dores lombares. Porém ao contrário do que a grande maioria da literatura científica cita sobre a lombalgia no que diz respeito à repercussão direta na qualidade de vida $(\mathrm{QV})$ dos seus portadores, os alunos que relataram esse tipo de dor não demonstram uma incapacidade física nas atividades de vida diárias (QVD) enquanto que a pesquisa demonstrou que o grupo que não possuía dor lombar apresentou uma QV pior, mas que não esta relacionada diretamente com a lombalgia nem com as posturas inadequadas decorrente da profissão.

\section{Referências Bibliográficas}

ANDRADE, Sandra Cristina.; ARAÚJO, Aurelan Geocarde Ribeiro.; VILAR, Maria José Pereira." Escola de Coluna": Revisão Histórica e Sua Aplicação na Lombalgia Crônica. Revista Brasileira de Reumatologia. v. 45, n. 5, p. 224-225, 2005.

BACHIEGA, Joanna Carolina. Sintomas de Distúrbios Osteomusculares Relacionados à Atividade de Cirurgiões-Dentistas Brasileiros. 2009. Dissertação (Mestrado) - Universidade Nove de Julho. São Paulo, 2009.

CASARIN, Cezar A. S.; CARIA, Paulo H. P. Comportamento Muscular Durante Diferentes Práticas Odontológicas. Ciências odontológicas Brasileira pag. 64-70. 2008

GARCIA, Patrícia Petromilli N S.; CAMPOS, Juliana Álvares Duarte Bonini.; ZUANON, Ângela Cristina Cilense. Avaliação Clínica das Posturas de Trabalho Empregadas na Clínica de Odontopediatria por Estudantes de Odontologia. Pesquisa Brasileira em Odontopediatria e Clínica Integrada João Pessoa, Vol. 8, Núm.1, p.31-37, 2008

GRAÇA, Claudia Cerqueira.; ARAÚJO, Tânia Maria.; SILVA, Cruiff Emerson Pinto. Desordens Musculoesqueléticas em Cirurgiões-Dentistas. Sitientibus, Feira de Santana, n 34, p. 71-86, 2006.

LIMA, Valquíria. Ginástica Laboral: Atividade Física no Ambiente De Trabalho: Ginastica Laboral Compensatoria, Ergonomia e Equipe Multidisciplinar. $3^{\mathrm{a}}$ ed. São Paulo: Phorte. 2007.

OCARINO, J. M.; GONÇALVES. G. G. P.; VAZ. D. V.; CABRAL. A. A. V.; PORTO. J. V.; SILVA. M. T. Correlação de um questionário de desempenho funcional e testes de capacidade física em Pacientes Com Lombalgia. Revista Brasileira de Fisioterapia. v.13, n 4, p. 345-347, 2009.

PRADO, Rejane Rossi. Avaliação da Qualidade de Vida na Indústria do Vestuário: O Caso das Costureiras Portadoras de Lombalgias. 2006. Dissertação (Mestrado) Universidade Estadual Paulista. Faculdade de Engenharia, Bauru, 2006. 
Rhobert Gomes Frauches; Walace Érick de Medeiros Moura; Júlio Corrêa Campos; Silvani Barreto Assumpção Cardoso \& Juliano Silva França

Incidência de Lombalgia nos Alunos do Oitavo Período do Curso de Odontologia da UNIG Campus V e a Sua Real Interferência na Qualidade de Vida

SOARES, Karoline Teles de Araújo,; VIESSER, Marcel Vidal,; RZNISKI, Tânia Aparecida Barbosa,; BRUM, Edison Paula. Eficácia de Um Protocolo de Exercícios Físicos em Pacientes Com Insuficiência Renal Crônica, Durante o Tratamento de Hemodiálise, Avaliado Pelo SF-36. Fisioterapia Movimento. v. 24, n. 1, p.135, 2011.

ZUFFO, Nívea Maria. Projeto de Intervenção Para Desequilíbrios Posturais e Sintomatologias Dolorosas Nos Cirurgiões Dentistas. Programa Postural. 2006 